\title{
Instructions for assembly
}

Creativity in science often emerges from paradox. The word 'crystal' captures the archetype of order - structures repeating perfectly through space, each elementary unit giving a complete description of the whole. Thirty years ago, the French mathematical physicist David Ruelle played imaginatively with paradox in a paper entitled 'Do turbulent crystals exist?' Turbulence? Crystals? Together?

By this time, Ruelle had already made seminal contributions to the theory of deterministic chaos. In the 1940s, Lev Landau, aiming for a general theory of the origins of turbulence, had suggested that its profound dynamic disorder develops progressively in a fluid as ever more Fourier modes get excited. In 1971, Ruelle, working with mathematician Floris Takens, showed that another 'scenario' for the transition to turbulence was actually much more likely in a mathematical sense. They showed that if an initially stable system undergoes four consecutive transitions to oscillatory behaviour, this is enough to create deterministic chaos and erratic unpredictable flow; later research reduced the number to three.

Loosely speaking, three independent oscillations are generically sufficient for chaos. In developing this idea - now known as the Ruelle-Takens-Newhouse route to chaos - Ruelle and Takens coined the famous term 'strange attractor'.

Chaos is a dynamical phenomenon that is, a process in time. Ruelle's paper with the paradoxical title simply asked if there might be analogues in space. If periodicity in time can dissolve into erratic chaos, why not in space? Could there be crystals, structured by deterministic rules, yet never repeating in space?

This was only a couple of years before the discovery of quasicrystals - ordered atomic solids with a quasiperiodic pattern in space. Ruelle (who assumed in his paper that quasicrystals would exist) was on to something. In the temporal domain, quasiperiodic time series lie between periodic and erratic structures; they define a boundary between periodic regularity and chaos. Quasicrystals play a similar role in the spatial domain. The rigid order of the classical crystal is as limiting as that of predictable, regular dynamics. What might we find on the far side?

Three decades later, we're finding out. As Julyan Cartwright and Alan Mackay argue

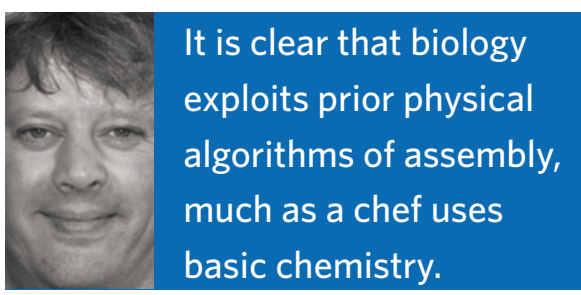

(Phil. Trans. R. Soc. A 370, 2807-2822; 2012), biology, physics and materials science now face the challenge of moving "beyond crystals" in a general way. The task is to bring some understanding to the world of disordered biological materials, and bioinspired synthetic systems. As they note, the word 'crystal' is still defined by the International Union of Crystallography as "a structure giving a diffraction pattern with discrete points". This is far too limiting.

The most famous aperiodic crystal is, of course, DNA, and there the varying structural pattern stores crucial information. But the role of information is more general, as virtually all structures in biology store information in one form or another, and reflect some essential biological function or purpose. At the simplest level, for example, we have membranes and micelles that divide the world into an inside and an outside, controlling flow between them. Biology is full of messy but still organized hierarchies and systems of systems running all the way from the atomic scale to the human - what draws them all together is the purposeful processing of information.

As Cartwright and Mackay propose, the relation between information and structure may offer a means of bringing some order to the world of messy bio-structures, or their analogues in synthetic materials. They introduce the 'assembly complexity' of a physical structure, this being a measure of how much information it holds. The idea works as an analogue of the famous Kolmogorov complexity-of-information theory. Whereas the Kolmogorov complexity of a numerical sequence is the length of the simplest algorithm capable of producing that sequence, the assembly complexity of a structure is given by the simplest physical algorithm capable of producing it.

How useful this idea might be in practice isn't yet clear. One shortcoming is that a realized process of assembly gives only an upper bound to the assembly complexity of the structure, as other simpler methods might exist. Even so, Cartwright and Mackay illustrate how the idea of assembly complexity frames some issues in an insightful way. For example, we may generally attribute to biology, and to the genetic machinery of biological organisms, the algorithmic capabilities behind all the various complex substances and structures they produce. The remarkably rich hierarchical structure of nacre (mother of pearl), seems to be all the product of the genetic machinery of molluscs. But this isn't quite right.

Nacre is an assembly of hexagonal platelets of aragonite, a form of calcium carbonate, stitched together into sheets that are then separated by further sheets of biopolymers such as chitin. The combination of brittle platelets and flexible biopolymer sheets makes the material remarkably strong and resistant to cracks. For this rich assembly, the mollusc genome takes some of the credit, but not all. From the information perspective, it is clear that biology actually exploits rich prior physical algorithms of assembly, much as a chef uses basic chemistry. Biology, as Cartwright and Mackay note, takes for granted "all the physics and chemistry of nucleation, growth dynamics, fluid dynamics, solid and liquid crystallization" and much more besides.

Hence, we should really attribute to the genome only the same information we do to a chef who exploits natural chemistry to produce a tasty soufflé, but who doesn't carry out the underlying physics and chemistry. Looking at information and where it resides in structures offers a way to penetrate the middle world between physicsbased materials science, approaching from the simple world of pure crystals, and biology, where complexity goes far beyond anything we can make artificially.

Yet we still don't have a strict answer to David Ruelle's original question: do turbulent crystals exist? For all the myriad amorphous structures known in biology, physics and modern chemistry, all seem to be metastable states or states of systems well out of equilibrium. Ruelle, in contrast, had envisaged turbulent crystals as the true physical ground states of a system at some finite temperature. It remains unknown whether such things may really exist.

Clearly, questions aren't valuable only if they can be answered. The act of trying is valuable in itself.

\section{MARK BUCHANAN}

